\title{
Medial Image of the Week: Palmar Erythema Multiforme
}

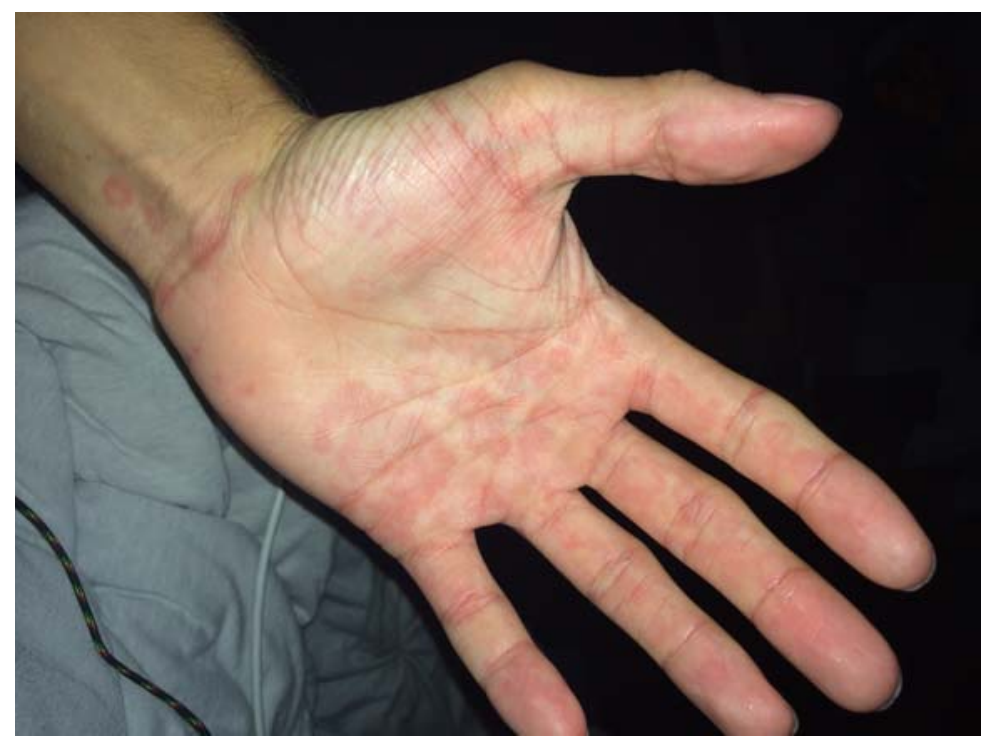

Figure 1. Palmar rash in a patient with acute pulmonary coccidioidomycosis resembling erythema multiforme.

An 18 year-old man presented for evaluation of abnormal chest imaging. Prior to moving to Tucson, Arizona three weeks before presentation, he was diagnosed with ankylosing spondylitis and was started on adalimumab (Humira). Approximately one week prior to presentation he developed a fever and cough. Over 3-4 days the cough worsened and he developed a palmar rash (Figure 1). He was seen in the emergency department and was started on levofloxacin. Prior to presentation in the pulmonary clinic his rash had resolved but the cough had persisted. Chest imaging showed airspace opacities within the left upper lobe with associated small left pleural effusion. Bronchoalveolar lavage revealed $57 \%$ eosinophils. Fungal cultures, bacterial cultures, and cytology were non-diagnostic. Coccidioides IgG antibody was negative but Coccidioides IgM antibody converted to positive, suggesting acute infection. The patient was started on oral fluconazole and clinically improved. Erythema nodosum and erythema multiforme are noted in as many as $25 \%$ of patients with acute pulmonary coccidioidomycosis. The rashes usually occur within a few days of infection and are considered a good prognostic sign (1).

Ryan Nahapetian, MD, MPH and Joshua Malo, MD Pulmonary, Allergy, Critical Care, \& Sleep Medicine University of Arizona, Tucson, AZ

\section{Reference}

1. Smith JA, Riddell J 4th, Kauffman CA. Cutaneous manifestations of endemic mycoses. Curr Infect Dis Rep. 2013;15(5):440-9. [CrossRef] [PubMed] 